\title{
Distributed Event-triggered Control for Reactive, Unbalanced and Harmonic Power Sharing in Islanded AC Microgrids
}

Lu, Jinghang; Zhao, Ming; Golestan, Saeed; Dragicevic, Tomislav; Pan, Xuewei; Guerrero, Josep M.

Published in:

IEEE Transactions on Industrial Electronics

Link to article, DOI:

10.1109/TIE.2021.3057018

Publication date:

2021

Document Version

Peer reviewed version

Link back to DTU Orbit

Citation (APA):

Lu, J., Zhao, M., Golestan, S., Dragicevic, T., Pan, X., \& Guerrero, J. M. (2021). Distributed Event-triggered Control for Reactive, Unbalanced and Harmonic Power Sharing in Islanded AC Microgrids. IEEE Transactions on Industrial Electronics, 69(2), 1548 - 1560. https://doi.org/10.1109/TIE.2021.3057018

\section{General rights}

Copyright and moral rights for the publications made accessible in the public portal are retained by the authors and/or other copyright owners and it is a condition of accessing publications that users recognise and abide by the legal requirements associated with these rights.

- Users may download and print one copy of any publication from the public portal for the purpose of private study or research.

- You may not further distribute the material or use it for any profit-making activity or commercial gain

- You may freely distribute the URL identifying the publication in the public portal 


\title{
Distributed Event-triggered Control for Reactive, Unbalanced and Harmonic Power Sharing in Islanded AC Microgrids
}

\author{
Jinghang Lu, Member, IEEE, Ming Zhao, Saeed Golestan, Senior Member, IEEE, \\ Tomislav Dragicevic, Senior Member, IEEE, Xuewei Pan, Member,IEEE, Josep Guerrero, \\ Fellow, IEEE,
}

\begin{abstract}
For several reasons, particularly due to the mismatch in the feeder impedance, accurate power sharing in islanded microgrids is a challenging task. To get around this problem, a distributed event-triggered power sharing control strategy is proposed in this paper. The suggested technique adaptively regulates the virtual impedances at both fundamental positive/negative sequence and harmonic frequencies and, therefore, accurately share the reactive, unbalanced, and harmonics powers among distributed generation (DG) units. The proposed method requires no information of feeder impedance and involves exchanging information among units at only eventtriggered times, which reduces the communication burden without affecting the system performance. The stability and inter-event interval are analyzed in this paper. Finally, experimental results are presented to validate the effectiveness of the proposed scheme.
\end{abstract}

Index Terms- Event-triggered control, distributed generation, droop control, microgrid, power sharing, virtual impedance.

\section{INTRODUCTION}

$\mathrm{D}$ ISTRIBUTED generation (DG) systems have been widely installed in the power distribution systems in recent years to meet the rising load demand with reduced negative effects on the environment and distribution infrastructures [1]. Several DG units are clustered together with local loads and energy storage systems leading to the concept of a microgrid [2]. Compared to the conventional distribution system, the microgrid can operate flexibly in either grid-connected mode or islanded mode to supply more reliable power to customers [3].

In the islanded mode of microgrids, an essential requirement is the proper active and reactive power sharing between DG units. Traditionally, active power-frequency $(P-\omega)$ and reactive

Manuscript received September 27th, 2020; revised January $1^{\text {st }}, 2021$; accepted January 21th, 2021. This work was supported by Shenzhen Overseas High Level Talent project (Peacock Plan). (corresponding author:Xuewei Pan)

Jinghang $\mathrm{Lu}$ is with School of Mechanical Engineering and Automation, Harbin Institute of Technology (Shenzhen), Shenzhen, China (Jinghang.lu@ieee.org)

Ming Zhao is with School of Mechanical Engineering and Automation, Harbin Institute of Technology (Shenzhen), Shenzhen, China (19s053064@stu.hit.edu.cn) power-voltage magnitude $(Q-E)$ based droop control has been widely applied in the system to regulate the power delivery [4] [5] [6]. However, a small mismatch in the line impedance may cause a noticeable error in the reactive power sharing using the $Q-E$ droop [8]. Some efforts to address this challenge of the droop method have been made in recent years (for example, see [7], [8]). These methods, however, still suffer from some inaccuracies, such as sensitivity to complex loads, among others. In addition, a fully decentralized control of gridconnected series inverters is firstly proposed to achieve the autonomous power balance in [9]. On the other hand, with growing power quality issues such as harmonics and voltage imbalance, which are attributable to the proliferation of nonlinear loads and supplying single-phase and/or unbalanced three-phase loads, the DG units are expected to properly share unbalanced power and harmonic powers. The traditional droop control, however, may not be able to achieve this objective, as the traditional droop is derived from the fundamental voltage model and does not take the unbalanced and harmonic powers into account. This issue can be especially serious if a mismatch in the line impedances exists.

In order to overcome these reactive power, unbalanced power, and harmonic power sharing issues, various methods have been proposed in the microgrid. These methods fall into two categories based on if the communication link is necessary, i.e., communication-less methods and communication-based methods. In the communication-less methods, several researchers directly add large virtual impedance in the control loops at the fundamental negative and harmonic frequencies $[10$, 11]. These methods, however, inevitably cause the unbalance and harmonic voltage drop even though the power sharing is improved. In order to overcome the aforementioned method's drawback, a virtual harmonic impedance and the $Z_{h}-H$ droop control scheme has been proposed in [12]. The exact line

Saeed Golestan is with the Department of Energy Technology, Aalborg University, Aalborg DK-9220, Denmark. (sgd@et.aau.dk)

Tomislav Dragicevic is with Electrical Engineering Department of Technical University of Denmark, Copenhagen, Denmark.(tomdr@elektro.dtu.dk)

Xuewei Pan is with School of Mechanical Engineering and Automation, Harbin Institute of Technology (Shenzhen), Shenzhen, China (davidpeterpan@163.com)

Josep M.Guerrero is with the Department of Energy Technology, Aalborg University, Aalborg DK-9220, Denmark. (email: joz@et.aau.dk) 
impedance is nevertheless required to design the coefficient of the $Z_{h}-H$ droop, which is quite challenging to implement in practice. In order to avoid the exact line impedance value, a small ac signal injection method is proposed by [13]; the injection and extraction of small ac signals still made the system complicated to implement.

On the other hand, the communication-based methods are proposed within the hierarchical control framework to share the reactive power, unbalanced power, and harmonic power [1417]. The power sharing strategy is usually calculated and implemented in the secondary control level, and then a command signal is sent to the primary control level through the communication links. The communication-based methods can be further classified into centralized control and distributed control strategy for the reactive, unbalanced, and harmonic power sharing. In [17], a microgrid centralized controller (MGCC) has been proposed to address the power sharing issue, where a compensation signal is sent from the MGCC to the DG units to regulate the virtual impedance. This method achieves good performance in the power sharing, but the regulation of the virtual impedance is achieved by adding the disturbance into the $P-\omega$ droop control. Therefore, the way of regulating the virtual impedance may not be recommended, as this disturbance causes active power temporary oscillation, and the critical load at the Point of Common Coupling (PCC) may be quite sensitive to this oscillation. A similar approach is proposed by [16], where the closed-loop poles are analyzed. It is found that this method may cause the closed-loop poles to move towards the imaginary axis. Furthermore, in a centralized control concept, the entire system relies on the MGCC and communication links. It implies that any failure in communication links may adversely affect the reliability of microgrid and the effectiveness of its control system. Moreover, when a new DG unit is connected to the microgrid system, a new communication link between the MGCC and the new DG unit needs be established, which may be challenging. This fact limits the scalability of microgrids built based on the centralized concept. To overcome the aforementioned limitations, the distributed control methods have been introduced in the microgrid systems to enhance the robustness and reliability of the system [18, 19]. In recent years, the consensus-based distributed control method has been widely adopted for microgrids $[15,20,21]$. In the consensus control scheme, only the local neighboring information is exchanged, and this method becomes more attractive to the microgrids due to its advantages in reducing consumption of the resources and increasing reliability [22]. Several attempts have been made to utilize a consensus-based distributed control strategy for microgrid. In [20], the reactive power sharing is achieved by utilizing the consensus algorithm. In addition, the consensus distributed algorithm proposed in [21] is used to share negative sequence current and compensate for the voltage unbalance at the PCC. Moreover, a similar method is proposed for unbalanced, and harmonic power sharing [15]. It should be mentioned that in the aforementioned distributed approaches, the information is exchanged periodically among the neighboring DG units, which indicates a large amount of data flows through the communication network. And this high data flowing through communication network may have some

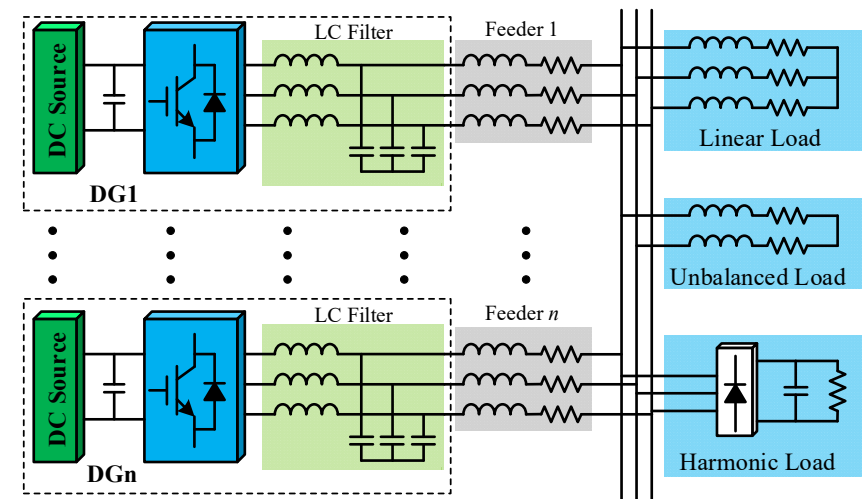

Fig. 1. Structure of islanded microgrid with $n$ DG units.

detrimental consequences, such as high costs and traffic congestion among others. As a result, designing a communication-saving strategy for the distributed control of microgrids is necessary [22, 23].

With the advance of communication technologies, the eventtriggered control methods have been increasingly applied in the microgrids [24-27]. Compared to the consensus-based distributed control, the event-triggered controllers only transmit information to its neighbors when the local state error exceeds a given threshold value, hence, the amount of communication data can be greatly reduced while maintaining an accurate control performance [28]. In [29], for instance, a distributed event-triggered algorithm is proposed to solve the economic dispatch issue for microgrid. In [25-27], the event-triggered control method is developed to restore the voltage and frequency among DG units. While the reactive power sharing with event-triggered control in a microgrid was studied in [24]. Although these works report the event-triggered control application in the microgrid systems, to the best of the authors' knowledge, the reactive, unbalanced, and harmonic power sharing issues by applying the event-triggered control strategy have not been discussed, and the stability of the event-triggered control for the power sharing has not been proved as well.

In response to the issues mentioned above, this paper proposes an event-triggered distributed control scheme for the reactive, unbalanced and harmonic power sharing in islanded microgrids. In order to reduce communication while guaranteeing the power sharing accuracy, the virtual impedance is adaptively regulated based on an event-triggered control strategy. The main contributions of this paper are summarized as follows:

1) A distributed event-triggered control strategy is proposed to achieve accurate reactive power, unbalanced power and harmonic power sharing in islanded microgrids. The proposed control strategy significantly reduces the utilization of communication while providing almost identical control performance to that of traditional periodic control scheme.

2) An event-triggered condition is designed and the stability is proved using Lyapunov function. The lower bound of inter-event time intervals are estimated. Thus, the Zeno behavior can be avoided. 

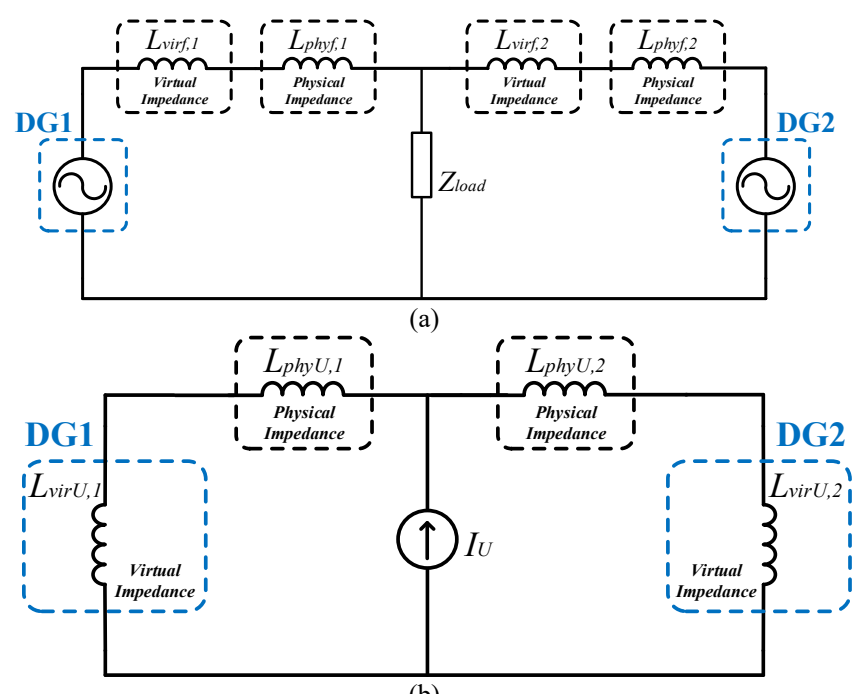

(b)

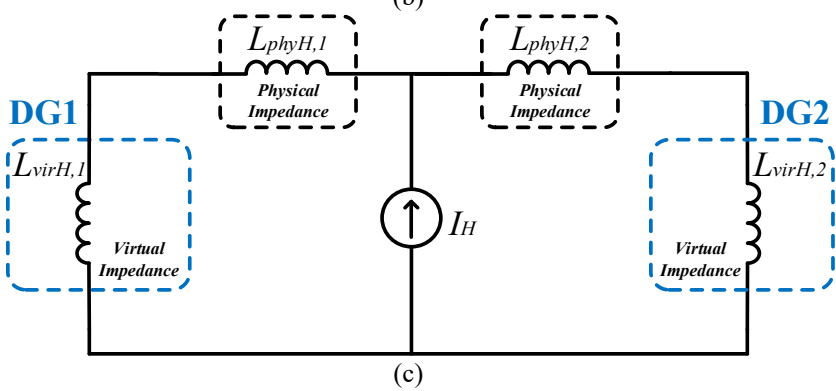

Fig. 2. Equivalent circuits of the microgrid at different frequencies. (a) Equivalent circuit at the fundamental positive-sequence frequency. (b) Equivalent circuit at fundamental negative-sequence frequency. (c) Equivalent circuit at harmonic frequencies.

3) The effectiveness of the proposed controllers is verified by experimental results, which demonstrate that the proposed approach can significantly reduce the communication burden while guaranteeing the power-sharing accuracy.

The rest of this paper is organized as follows. The structure of the microgrid system along with an analysis of the power sharing control and graph theory are introduced in Section II. Section III presents the proposed control strategy. The stability analysis based on Lyapunov function and inter-event interval analysis are presented in Section IV. The proposed method is validated by experiments in Section V. Finally, the conclusion is presented in Section VI.

\section{ISLANDED MICROGRID ANALYSIS}

\section{A. Principle of Droop Control}

Fig. 1 shows the structure of an islanded microgrid, where the DG units are interfaced to the microgrid through different feeders. Each DG unit is comprised of a DC source, an inverter, and an LC type filter. The microgrid also includes linear, unbalanced, and nonlinear/harmonic loads placed at the PCC.

The power sharing of DG units in islanded microgrids, as mentioned before, is often based on the conventional $P-\omega$ and $Q-E$ droop controllers in DG units, which can be expressed as

$$
\begin{aligned}
& \omega=\omega_{0}-m P \\
& E=E_{0}-n Q
\end{aligned}
$$

where $\omega_{0}$ and $E_{0}$ are the nominal values of the DG angular frequency and voltage magnitude; $m$ and $n$ are the droop coefficients; $P$ and $Q$ are the low-pass filtered active and reactive powers, respectively. The outputs of the droop control are fed to a reference generation unit, which generates the fundamental reference voltage $V_{\text {droop, } \alpha \beta}$ in the stationary reference frame for the DG unit.

A mismatch in DGs' feeders may adversely affect the stability and power sharing between DGs. To reduce the influence of feeder impedance, the virtual impedance $\left(Z_{v i r}\right)$ is introduced. Then, the voltage reference from the droop controller is modified as

$$
V_{\text {ref }, \alpha \beta}=V_{\text {droop }, \alpha \beta}-Z_{\text {vir }} i_{\alpha \beta}
$$

where $V_{\text {ref, } \alpha \beta}$ is the voltage reference considering virtual impedance and $i_{\alpha \beta}$ is the DG output current.

\section{B. Reactive, Unbalanced, and Harmonic Power Sharing Analysis}

To simplify the analysis, an islanded microgrid including two DG units with the equal power rating is considered. In addition, it is assume that the feeder impedances are inductive. Fig. 2 presents an equivalent circuit with two DG units. It can be seen from Fig. 2(a) that DG units are modeled by controlled voltage sources at the fundamental positive-sequence frequency (FPF). Meanwhile, the load is considered as a passive load. From Fig. 2(a), the DG equivalent impedance $L_{f, i}$ at the FPF becomes:

$$
L_{f, i}=L_{\text {phyf }, i}+L_{\text {virf }, i}
$$

where $L_{p h y f i}$ and $L_{v i r f, i}$ are the physical feeder impedance and virtual impedance at the FPF.

In addition, the equivalent circuit at the fundamental negative sequence frequency (FNF) is illustrated in Fig. 2(b). The unbalanced load is modeled as a current source $\left(I_{U}\right)$ [30]. From Fig.2(b), the DG equivalent fundamental negative sequence impedance $L_{U, i}$ becomes:

$$
L_{U, i}=L_{p h y U, i}+L_{v i r U, i}
$$

where $L_{p h y, i}$ and $L_{v i r U, i}$ are the physical feeder impedance and virtual impedance at the fundamental negative-sequence frequency, respectively.

Similarly, the equivalent impedance at harmonic frequencies is given in Fig. 2(c) as

$$
L_{H, i}=L_{p h y H, i}+L_{v i r H, i}
$$

where $L_{p h y, i}$ and $L_{v i r H, i}$ are, respectively, the physical feeder impedance and virtual impedance at harmonic frequencies.

To achieve accurate power sharing, the same equivalent impedance must be controlled in both DG units. Fortunately, as it can be seen in (4)-(6), the virtual impedance provides an efficient way for controlling the DG equivalent impedance at a given frequency. Therefore, by regulating the virtual impedances at fundamental positive and negative sequences and at harmonic frequencies, proper reactive/unbalanced/harmonic power sharing can be achieved.

Note that the DG units are assumed to be connected to the PCC with inductive physical feeders in the islanded microgrid. This assumption is reasonable because series coupling inductors are normally required in the DG units to ensure the 


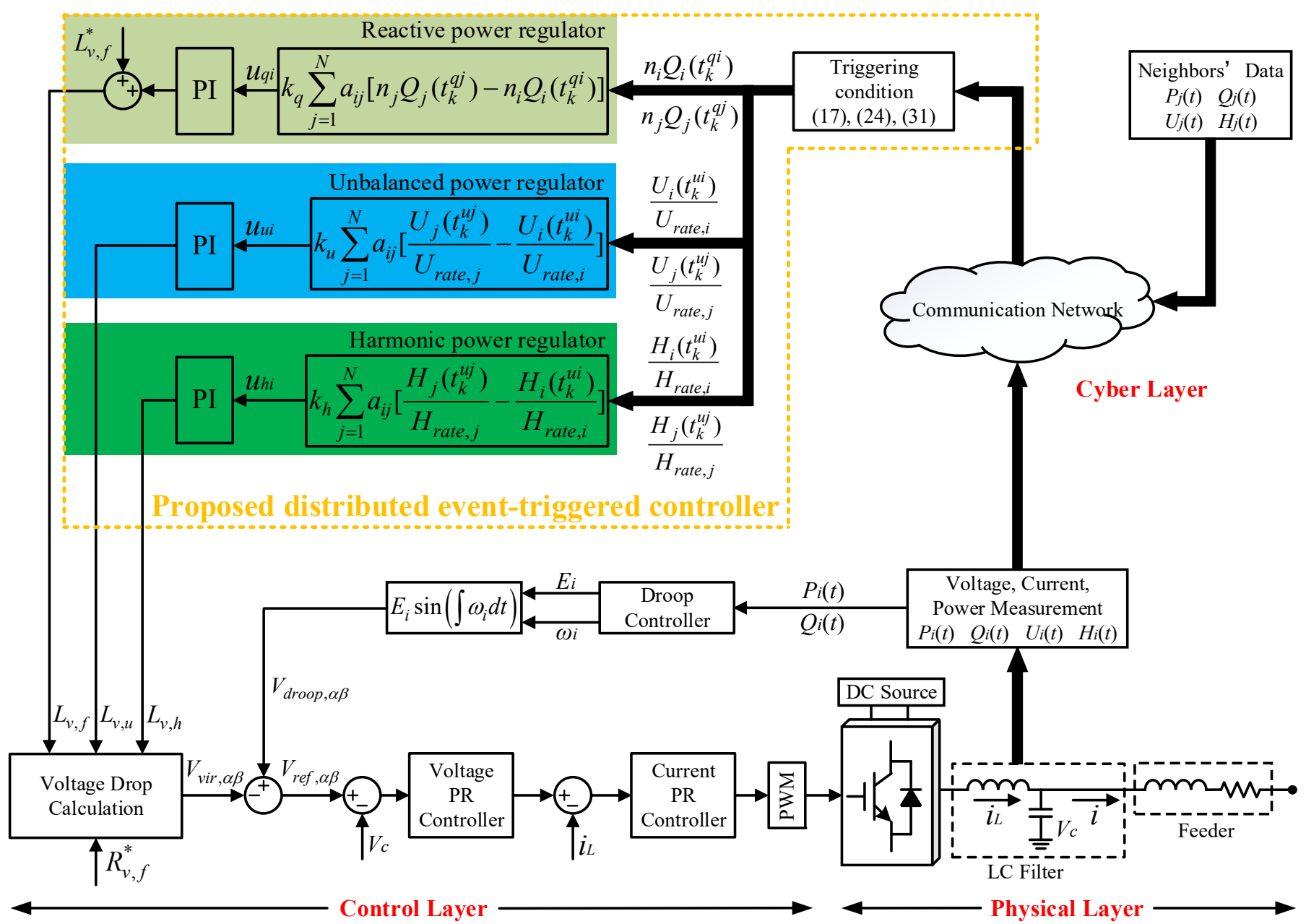

Fig. 3. Overall control diagram of the proposed distributed control scheme.

stability of the power sharing. In addition, DG units are often interconnected to the distribution system with isolation transformers, which have highly inductive leakage impedance. Finally, even when DG units are interfaced to the PCC with resistive line impedance, the fixed value virtual inductive impedance can be added and pre-activated in the control scheme. Therefore, if the preactivated virtual inductive impedance is properly designed, the DG equivalent impedance can be inductive.

\section{Communication Network}

The communication topology of a microgrid is depicted as a graph $\mathcal{G}=(\mathcal{V}, \mathcal{E}, A)$, which consists of a set of nodes $\mathcal{V}=$ $\left(v_{1}, v_{2}, \ldots, v_{N}\right)$, where $v_{i}$ represents DG $i$, a set of edges $\mathcal{E} \subseteq$ $\mathcal{V} \times \mathcal{V}$, and the adjacency matrix $A=\left[a_{i j}\right] \in \mathbb{R}^{N \times N} .\left(v_{j}, v_{i}\right) \in$ $\mathcal{E}$ denotes an edge, which means node $j$ can transmit its own information to node $i$. The graph $\mathcal{G}$ is said to be undirected if for all edges $\left(v_{j}, v_{i}\right) \in \mathcal{E},\left(v_{i}, v_{j}\right) \in \mathcal{E}$. The neighboring set of $i$ is presented as $N_{i}=\left\{v_{j} \in \mathcal{V} \mid\left(v_{j}, v_{i}\right) \in \mathcal{E}, i \neq j\right\}$. The elements $a_{i j}=1$ if $\left(v_{j}, v_{i}\right) \in \mathcal{E}$; otherwise, $a_{i j}=0$. The degree matrix $D=\operatorname{diag}\left\{d_{1}, \ldots, d_{N}\right\}$ is a diagonal matrix with $d_{i}=\left|N_{i}\right|$. The Laplacian matrix $L$ is defined as $L=D-A$. A path from node $i$ to node $j$ is a sequence of edges, belong to $\mathcal{E}$, which can be expressed as $\left\{\left(v_{i}, v_{k}\right), \ldots,\left(v_{k}, v_{j}\right)\right\}$. If there exists a path between any nodes, the graph is said to be connected [31].

\section{Proposed Control Scheme}

In this paper, a distributed event-triggered control scheme is proposed to adaptively regulate the virtual impedance. The overall control diagram is shown in Fig. 3, which mainly includes the primary control, distributed adaptive virtual impedance controller as well as a communication layer. With the proposed method, proper reactive, unbalanced, and harmonic power sharing can be achieved by, respectively, regulating the virtual impedances at fundamental positive sequence, fundamental negative sequence, and selected harmonic frequencies without any knowledge of line impedance. Note that the proposed approach is fully distributed. Each controller requires only the local and its neighbors' information to achieve power sharing performance. Note also that the controller transmits its information to neighbors only at event-triggered times. Therefore, the communication burden is considerably reduced compared to the traditional periodic communication way. 


\section{A. Power Calculation}

The reactive, unbalanced, and harmonic power calculation involves extracting the fundamental-frequency positive- and negative-sequence components and some concerned harmonic components. The multiple second-order generalized integrators (SOGI)-based frequency-locked loop is used for this purpose [32]. With the detected current and voltage components, the DG output active power $P$, reactive power $Q$, unbalanced power $U$ and harmonic power $H$ can be calculated as

$$
\begin{gathered}
P=\frac{3}{2} \frac{\omega_{c}}{s+\omega_{c}}\left(v_{C \alpha} i_{f \alpha}^{+}+v_{C \beta} i_{f \beta}^{+}\right) \\
Q=\frac{3}{2} \frac{\omega_{c}}{s+\omega_{c}}\left(v_{C \beta} i_{f \alpha}^{+}-v_{C \alpha} i_{f \beta}^{+}\right) \\
U=\frac{3 E_{0}}{2} \frac{\omega_{c}}{s+\omega_{c}} \sqrt{\left(I_{f \alpha}^{-}\right)^{2}+\left(I_{f \beta}^{-}\right)^{2}} \\
H=\frac{3 E_{0}}{2} \frac{\omega_{c}}{s+\omega_{c}} \sqrt{\left(I_{5 \alpha}^{-}\right)^{2}+\left(I_{5 \beta}^{-}\right)^{2}+\left(I_{7 \alpha}^{+}\right)^{2}+\left(I_{7 \beta}^{+}\right)^{2}}
\end{gathered}
$$

Where $E_{0}$ is the nominal voltage, $\omega_{c}$ is the cut-off frequency of LPF. $v_{C \alpha}$ and $v_{C \beta}$ are the measured DG voltage in the stationary reference frame. $i_{f \alpha}^{+}$and $i_{f \beta}^{+}\left(i_{f \alpha}^{-}\right.$and $\left.i_{f \beta}^{-}\right)$are the fundamental positive (negative) sequence current. $i_{7 \alpha}^{+}$and $i_{7 \beta}^{+}$.

\section{B. Controller Design}

A distributed event-triggered adaptive virtual impedance controller is designed in this part.

1) Reactive power sharing: Construct the state model of the reactive power sharing control as

$$
n_{i} \dot{Q}_{i}=u_{q i}
$$

where $u_{q i}$ denotes the input for reactive power controller.

A distributed controller can be constructed as

$$
u_{q i}(t)=k_{q} z_{q i}(t)
$$

where $k_{q}$ is a proportional gain and $z_{q i}(\mathrm{t})$ is defined as

$$
z_{q i}(t)=\sum_{j=1}^{N} a_{i j}\left[n_{j} Q_{j}(t)-n_{i} Q_{i}(t)\right]
$$

Under the proposed event-triggered control scheme, (13) is redefined as

$$
z_{q i}(t)=\sum_{j=1}^{N} a_{i j}\left[n_{j} Q_{j}\left(t_{k}^{q j}\right)-n_{i} Q_{i}\left(t_{k}^{q i}\right)\right]
$$

The state measurement error is defined as

$$
e_{q i}(t)=n_{i} Q_{i}\left(t_{k}^{q i}\right)-n_{i} Q_{i}(t), t \in\left[t_{k}^{q i}, t_{k+1}^{q i}\right)
$$

Note that the measurement error $\left\|e_{q i}(t)\right\|$ is the deviation between the latest triggering time state and the real-time state. When $\left\|e_{q i}(t)\right\|$ reaches a predefined threshold, the event is triggered, the state estimate is equal to the actual value and $\left\|e_{q i}(t)\right\|$ is reset to zero. During the event time interval, no communication is required.

Theorem 1: Assume that the communication topology $\mathcal{G}$ is undirected and connected. Then, the controller in (12) and (14) can achieve reactive power sharing if the event-triggered time is defined as follows [26]:

$$
t_{k}^{q i}=\inf \left\{t>t_{k-1}^{q i} \mid f_{q i}(t)=0\right\}
$$

where the triggering function $f_{q i}(\mathrm{t})$ can be defined as

$$
f_{q i}(t)=\left\|e_{q i}(t)\right\|^{2}-\frac{\sigma_{q}\left(1-\alpha_{q}\left|N_{i}\right|\right)}{\left|N_{i}\right| / \alpha_{q}}\left\|z_{q i}(t)\right\|^{2}
$$

where $0<\sigma_{\mathrm{q}}<1,0<\alpha_{\mathrm{q}}<1 /\left|N_{i}\right|$.
If the reactive power is not shared accurately by each DG unit, the sharing error generated by the local controllers is utilized to adaptively regulate the virtual inductance as follows:

$$
L_{v, f}=L_{v, f}^{*}-G_{q}(s) u_{q i}
$$

where $L_{v, f}^{*}$ is the static virtual impedance at the fundamental positive sequence, which is used to ensure the fundamental equivalent impedance is inductive, $L_{v, f}$ is adaptively regulated by an integral controller to eliminate the reactive power sharing error; and $G_{q}(s)$ is a proportional integral (PI) controller.

2) Unbalanced power sharing: Construct the state model of the unbalanced power sharing control as follows:

$$
\frac{\dot{U}_{i}}{U_{\text {rate }, i}}=u_{u i}
$$

where $U_{\text {rate }, i}$ is the unbalanced power rating of the $i$ th DG units. $u_{u i}$ denotes the input for unbalanced power controller.

A distributed controller can be construct as

$$
u_{u i}(t)=k_{u} z_{u i}(t)
$$

where $k_{u}$ is a proportional gain and $z_{u i}(\mathrm{t})$ is defined as

$$
z_{u i}(t)=\sum_{j=1}^{N} a_{i j}\left[\frac{U_{j}\left(t_{k}^{u j}\right)}{U_{\text {rate }, j}}-\frac{U_{i}\left(t_{k}^{u i}\right)}{U_{\text {rate }, i}}\right]
$$

The state measurement error is calculated from

$$
e_{u i}(t)=\frac{U_{i}\left(t_{k}^{u i}\right)}{U_{\text {rate }, i}}-\frac{U_{i}(t)}{U_{\text {rate }, i}}, t \in\left[t_{k}^{u i}, t_{k+1}^{u i}\right)
$$

Theorem 2: Assume that the communication topology $\mathcal{G}$ is undirected and connected. Then, the controller in (20) and (21) can achieve unbalanced power sharing if the event-triggered time is defined as follows [26]:

$$
t_{k}^{u i}=\inf \left\{t>t_{k-1}^{u i} \mid f_{u i}(t)=0\right\}
$$

where the triggering function $f_{u i}(\mathrm{t})$ can be defined as

$$
f_{u i}(t)=\left\|e_{u i}(t)\right\|^{2}-\frac{\sigma_{u}\left(1-\alpha_{u}\left|N_{i}\right|\right)}{\left|N_{i}\right| / \alpha_{u}}\left\|z_{u i}(t)\right\|^{2}
$$

where $0<\sigma_{\mathrm{u}}<1,0<\alpha_{\mathrm{u}}<1 /\left|N_{i}\right|$.

In a similar manner, the virtual impedance at fundamental negative sequence $L_{v, u}$ is adaptively regulated to remove the unbalanced power sharing error as

$$
L_{v, u}=-G_{u}(s) u_{u i}
$$

where $G_{u}(s)$ is a PI controller.

3) Harmonic power sharing: Construct the state model of the harmonic power sharing control as follows:

$$
\frac{\dot{H}_{i}}{H_{\text {rate }, i}}=u_{h i}
$$

where $H_{\text {rate }, i}$ are the harmonic power rating of the $i$ th DG unit. $u_{q i}$ denotes the input for harmonic power controller.

A distributed controller can be constructed as

$$
u_{h i}(t)=k_{h} z_{h i}(t)
$$

where $k_{h}$ is a proportional gain and $z_{h i}(t)$ are defined as

$$
z_{h i}(t)=\sum_{j=1}^{N} a_{i j}\left[\frac{H_{j}\left(t_{k}^{h j}\right)}{H_{\text {rate }, j}}-\frac{H_{i}\left(t_{k}^{h i}\right)}{H_{\text {rate }, i}}\right]
$$

The state measurement error is defined as

$$
e_{h i}(t)=\frac{H_{i}\left(t_{k}^{h i}\right)}{H_{\text {rate }, i}}-\frac{H_{i}(t)}{H_{\text {rate }, i}}, t \in\left[t_{k}^{h i}, t_{k+1}^{h i}\right]
$$

Theorem 3: Assume that the communication topology $\mathcal{G}$ is undirected and connected. Then, the controller in (27) and (28) can achieve harmonic power sharing if the event-triggered time is defined as follows [26]:

$$
t_{k}^{h i}=\inf \left\{t>t_{k-1}^{h i} \mid f_{h i}(t)=0\right\}
$$




$$
f_{h i}(t)=\left\|e_{h i}(t)\right\|^{2}-\frac{\sigma_{h}\left(1-\alpha_{h}\left|N_{i}\right|\right)}{\left|N_{i}\right| / \alpha_{h}}\left\|z_{h i}(t)\right\|^{2}
$$

where $0<\sigma_{\mathrm{h}}<1,0<\alpha_{\mathrm{h}}<1 /\left|N_{i}\right|$.

Finally, the virtual impedance at harmonic frequency $L_{v, h}$ is adaptively regulated to remove the harmonic power sharing error as

$$
L_{v, h}=-G_{h}(s) u_{h i}
$$

where $G_{h}(s)$ is a PI controller.

Once the virtual impedance is determined, its corresponding voltage drops in the stationary reference frame can be calculated as follows:

$$
\begin{array}{r}
V_{f, \alpha \beta}=R_{v, f}^{*} i_{f \alpha}^{+}-\omega L_{v, f} i_{f \beta}^{+} \\
V_{U, \alpha \beta}=-\omega L_{v, u} i_{f \beta}^{-} \\
V_{H, \alpha \beta}=-\omega L_{v, h} i_{h \beta} .
\end{array}
$$

Then, the voltage reference for the double-loop voltage controller is obtained as

$$
\begin{aligned}
V_{\text {ref }, \alpha \beta} & =V_{\text {droop }, \alpha \beta}-V_{v i r, \alpha \beta} \\
& =V_{\text {droop }, \alpha \beta}-V_{f, \alpha \beta}-V_{U, \alpha \beta}-V_{H, \alpha \beta} .
\end{aligned}
$$

4) Double-loop voltage control: The outer loop voltage controller is implemented to regulate the output capacitor's voltage. The inner loop current controller is nested inside the voltage control loop to regulate the inverter side current. The controllers for voltage and current regulation are expressed as:

$$
\begin{gathered}
G_{V}(s)=k_{p V}+\frac{k_{V 1} s}{s^{2}+\left(\omega_{0}\right)^{2}}+\sum_{h=5,7} \frac{k_{V h} s}{s^{2}+\left(h \omega_{0}\right)^{2}} \\
G_{I}(s)=k_{p I}+\frac{k_{r I} s}{s^{2}+\left(\omega_{0}\right)^{2}}
\end{gathered}
$$

where $k_{p V}$ and $k_{p I}$ are the proportional terms, $k_{V 1}$ and $k_{r I}$ are the resonant term coefficients at $\omega_{o}=2 \pi \times 50 \mathrm{rad} / \mathrm{s} . k_{V h}$ is the resonant coefficient term for the $h^{\text {th }}$ harmonics $\left(5^{\text {th }}, 7^{\text {th }}\right)$. The inner current loop is designed to provide sufficient damping and protect the inductor's current from overcurrent.

\section{STABILITY ANALYSIS}

In this section, we first utilize the Lyapunov function to prove the stability of the proposed method, taking Theorem 1 as an example. Then, to avoid infinite event-triggered instants in a limited time, the lower bound of inter-event interval is analyzed, and Zeno behavior can be excluded [33], [34].

\section{A. Proof of Theorem 1}

To simplify the proof, we omit the subscript $Q$, abbreviate $x(\mathrm{t})$ to $x$, and denote $q_{i}=n_{i} Q_{i}$. Combining (11), (12), (14) and (15), the overall system dynamics can be written as

$$
\dot{q}=-k L(q+e)
$$

where $q=\left[q_{1}, q_{2}, \ldots, q_{N}\right]^{T}, e=\left[e_{1}, e_{2}, \ldots, e_{N}\right]^{T}$. Similarly, we have

where $z=\left[z_{1}, z_{2}, \ldots, z_{N}\right]^{T}$.

$$
z=-L(q+e)
$$

Considering the Lyapunov function candidate

$$
V=\frac{1}{2} q^{T} L q
$$

Then, the time derivative of (42) becomes

$$
\dot{V}=q^{T} L \dot{q}
$$

$$
\dot{V}=-k q^{T} L^{2}(q+e)
$$

Placing (41) into the upper equation in (44) yields

$$
\dot{V}=-k z^{T} z-k e^{T} L z=-k\|z\|^{2}-k e^{T} L z
$$

Expanding (45), we get

$$
\begin{aligned}
V & =-k \sum_{i=1}^{N} z_{i}^{2}-k \sum_{i=1}^{N} \sum_{j \in N_{i}} e_{i}\left(z_{i}-z_{j}\right) \\
& =-k \sum_{i=1}^{N} z_{i}^{2}-k \sum_{i=1}^{N}\left|N_{i}\right| e_{i} z_{i}+k \sum_{i=1}^{N} \sum_{j \in N_{i}} e_{i} z_{i}
\end{aligned}
$$

Using the inequality

$$
|x y| \leq \frac{1}{2 \alpha} x^{2}+\frac{\alpha}{2} y^{2}, \alpha>0
$$

The equation in (46) can be bounded by

$$
\begin{aligned}
\dot{V} \leq & -k \sum_{i=1}^{N} z_{i}^{2}+k \sum_{i=1}^{N} \frac{1}{\alpha}\left|N_{i}\right| e_{i}^{2}+k \sum_{i=1}^{N} \frac{\alpha}{2}\left|N_{i}\right| z_{i}^{2} \\
& +k \sum_{i=1}^{N} \sum_{j \in N_{i}} \frac{\alpha}{2} z_{j}^{2}
\end{aligned}
$$

Since the undirected graph $\mathcal{G}$ is symmetric, by interchanging the indices of the last term, we get

$$
\sum_{i=1}^{N} \sum_{j \in N_{i}} \frac{\alpha}{2} z_{j}^{2}=\sum_{i=1}^{N} \sum_{j \in N_{i}} \frac{\alpha}{2} z_{i}^{2}=\sum_{i=1}^{N} \frac{\alpha}{2}\left|N_{i}\right| z_{i}^{2}
$$

So that

$$
\dot{V} \leq-k \sum_{i=1}^{N}\left(1-\alpha\left|N_{i}\right|\right) z_{i}^{2}+k \sum_{i=1}^{N} \frac{1}{\alpha}\left|N_{i}\right| e_{i}^{2}
$$

Assuming that

$$
0<\alpha<1 /\left|N_{i}\right|
$$

Then, if the following condition holds

We get

$$
e_{i}^{2} \leq \frac{\sigma\left(1-\alpha\left|N_{i}\right|\right)}{\left|N_{i}\right| / \alpha} z_{i}^{2}, \quad 0<\sigma<1
$$

$$
\dot{V} \leq 0
$$

Thus, the triggering function defined in (17) can ensure $q(\mathrm{t})$ is stable. The proof is completed.

\section{B. Inter-event Interval Analysis}

Theorem 4: Assume that the communication topology $\mathcal{G}$ is undirected and connected. Consider the system in (11) with the controller in (12) and (14). If the triggering function is defined as (18), then the inter-event interval is lower bounded by a positive constant $\tau$.

Proof: Considering the following time derivative

$$
\frac{d}{d t} \frac{\|e\|}{\|z\|}=\frac{d}{d t} \frac{\left(e^{T} e\right)^{\frac{1}{2}}}{\left(z^{T} z\right)^{\frac{1}{2}}}=\frac{e^{T} \dot{e}}{\|e\|\|z\|}-\frac{\|e\|\left(z^{T} \dot{z}\right)}{\|z\|^{3}}
$$

According to (15), the derivative of state measurement error $e$ can be written as

$$
\dot{e}=-\dot{q}
$$

Then the derivative of $z$ in (41) is

$$
\dot{z}=-L(\dot{q}+\dot{e})=0
$$

Placing (55), (56) into (54) yields

$\frac{d}{d t} \frac{\|e\|}{\|z\|}=-\frac{e^{T} \dot{e}}{\|e\|\|z\|}-\frac{\|e\|\left(z^{T} \dot{z}\right)}{\|z\|^{3}} \leq \frac{\|e\|\|\dot{q}\|}{\|e\|\|z\|}+\frac{\|e\|\|z\|\|\dot{z}\|}{\|z\|^{3}}$

Combining (40), then (43) can be written as 

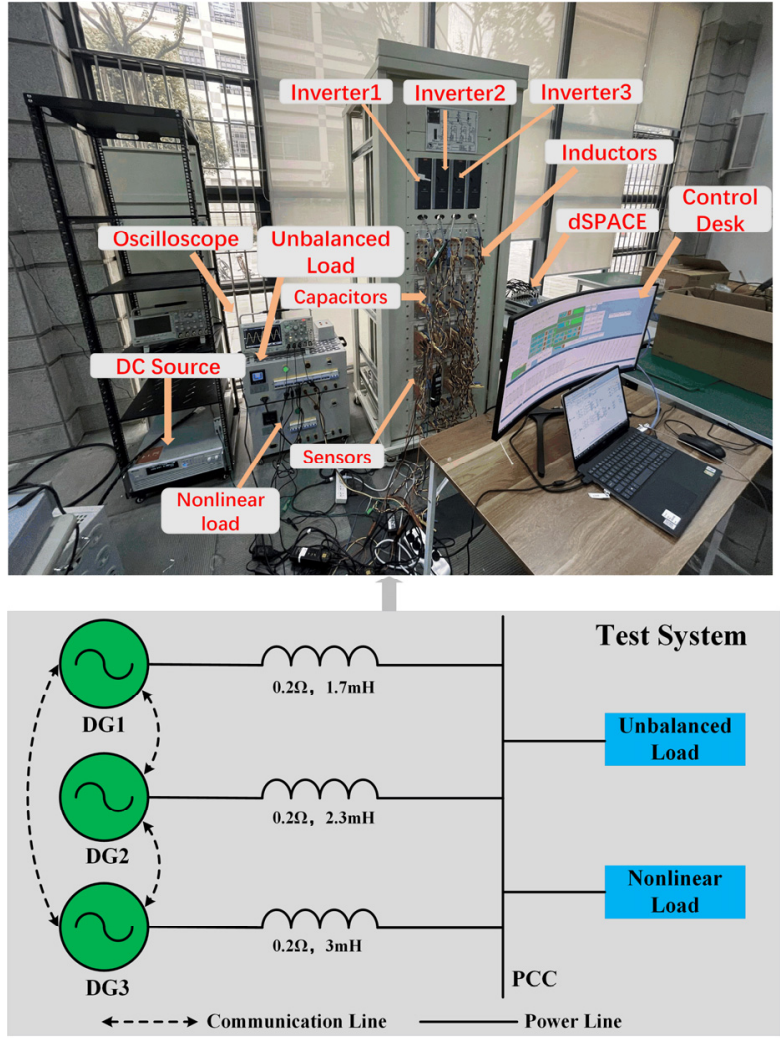

Fig. 4. Microgrid setup in the experiment

$$
\begin{aligned}
& =\frac{\|\dot{q}\|}{\|z\|}+\frac{\|e\|\|\dot{z}\|}{\|z\|^{2}} \\
& =\frac{\|\dot{q}\|}{\|z\|}
\end{aligned}
$$

Placing the derivative of $q$ in (40) into (57), we get

$$
\frac{d}{d t} \frac{\|e\|}{\|z\|} \leq k \frac{\|L\|(\|q\|+\|e\|)}{\|z\|}
$$

Note that $L$ is reversible; combining (58) with (41) yields

$$
\frac{d}{d t} \frac{\|e\|}{\|z\|} \leq k \frac{\|L\|(\|q\|+\|e\|)}{\|z\|}
$$

Thus, $\|e\| /\|z\|$ is upper bounded by

$$
\frac{\|e\|}{\|z\|} \leq \phi\left(t, \phi_{0}\right)
$$

where $\phi\left(t, \phi_{0}\right)$ is the solution of the following differential equations:

Thus, we have

$$
\left\{\begin{array}{l}
\dot{\phi}=k(2\|L\| \phi+1) \\
\phi\left(0, \phi_{0}\right)=\phi_{0}
\end{array}\right.
$$

$$
\phi(\tau, 0)=\frac{1}{2\|L\|}\left(e^{2 k\|L\| \tau}-1\right)
$$

Denoting

$$
h=\arg \max _{i}\left\|z_{i}\right\|
$$

Because $\left|e_{i}\right| \leq\|e\|$ holds, we have

$$
\frac{\left|e_{h}\right|}{\left|z_{h}\right|} \leq \frac{\|e\|}{\left|z_{h}\right|} \leq \frac{N\|e\|}{\|z\|}
$$

Placing (61) and the triggering function in (17) into (64), we have

$$
\sqrt{\frac{\sigma\left(1-\alpha\left|N_{i}\right|\right)}{\left|N_{i}\right| / \alpha}} \leq \frac{N}{2\|L\|}\left(e^{2 k\|L\| \tau}-1\right)
$$

$$
\tau \geq \frac{1}{2 k\|L\|} \ln \left[\frac{2\|L\|}{N} \sqrt{\frac{\sigma\left(1-\alpha\left|N_{i}\right|\right)}{\left|N_{i}\right| / \alpha}}+1\right]
$$

Thus, the inter-event interval is lower bound, and Zeno behavior is avoided. The proof is completed.

\section{EXPERIMENTAL RESULTS}

To demonstrate the effectiveness of the proposed control technique, experiments are provided at the HIT-Shenzhen microgrid laboratory. The microgrid setup, as shown in Fig. 4, includes three DG units at the same power rating supplying unbalance/nonlinear loads connected to PCC. Each DG unit consists of a Danfoss FC302 converter with $L C$ type filters. The dSPACE microlabbox is chosen to be the controller in the experiment. The communication links among three DG units are bidirectional. The physical system parameters and control ones are listed in Table I.

\section{A. Case-1: Unbalanced Load}

In this section, only an unbalanced RL load is connected to PCC. The PCC voltage is $120 \mathrm{~V}$ rms, as shown in Fig. 5. Fig. 6 shows the power sharing performance of the proposed method. To save space, the output active powers are not shown. At $t<t_{1}$ (i.e., stage 1), the traditional droop controller is applied. It can be observed that the reactive and unbalanced powers are shared improperly among parallel units. This result was expected as there is a mismatch in line impedances. At $t=t_{1}$, the proposed control mechanism is activated. Thanks to the action of the proposed control strategy, the reactive and unbalanced powers are converged to a common value after a short while. Recall that the proposed controller adaptively regulates the virtual impedances to compensate for the line impedance mismatches and achieve proper power sharing among parallel units.

The corresponding phase A current waveforms of the three DG units are shown in Fig. 7. Fig. 7(a) shows phase A current of three DG units using the traditional droop method. It obviously illustrates that the current magnitudes of phase A in three DG units are not the same. This is because of the unequal power sharing in the microgrid. After the proposed method is activated, the phase A current of the three DG units are almost

TABLE I

EXPERIMENTAL PARAMETERS OF THE MICROGRID

\begin{tabular}{clc}
\hline \hline Symbol & \multicolumn{1}{c}{ Quantity } & Nominal Value \\
\hline$V_{D C}$ & DC voltage & $400 \mathrm{~V}$ \\
$f_{s w}$ & Switching frequency & $10 \mathrm{kHz}$ \\
$\omega_{0}$ & Nominal frequency & $2 \pi \times 50 \mathrm{rad} / \mathrm{s}$ \\
$E_{0}$ & Nominal voltage & $120 \mathrm{Vrms}$ \\
$L_{f} / C_{f}$ & LC filter & $1.8 \mathrm{mH} / 25 \mu \mathrm{F}$ \\
$P_{U} / Q_{U}$ & Unbalanced load & $5 \mathrm{~kW} / 4 \mathrm{kVar}$ \\
$\mathrm{C}_{\mathrm{HL}} / \mathrm{R}_{\mathrm{HL}}$ & Nonlinear load & $4700 \mu \mathrm{F} / 72 \Omega$ \\
$R_{v, f}^{*} / L_{v, f}^{*}$ & Static virtual impedance & $0.5 \Omega / 1.5 \mathrm{mH}$ \\
$m$ & $P-\omega$ droop coefficient & 0.0002 \\
$n$ & $Q-E$ droop coefficient & 0.0005 \\
$\omega_{c}$ & LPF cut-off frequency & $4 \pi \mathrm{rad} / \mathrm{s}$ \\
$K_{p V} / K_{V I}$ & Voltage PR controller & $0.04 / 100$ \\
$K_{V I} / K_{V 7}$ & & $50 / 50$ \\
$K_{p l} / K_{r I}$ & Current PR controller & $5.7 / 500$ \\
$K_{p Q} / K_{I Q}$ & Reactive PI controller & $0.001 / 0.03$ \\
$K_{p U} / K_{I U}$ & Unbalance PI controller & $0.001 / 0.02$ \\
$K_{p H} / K_{I H}$ & Harmonic PI controller & $0.002 / 0.05$ \\
\hline \hline
\end{tabular}


identical, as shown in Fig. 7(b).

Fig. 8 validates the plug-and-play ability of the proposed method. At $t<t_{1}$ (i.e., stage 1), all three DG units supply the power to the unbalanced load connecting to the PCC. Fig. 8(a) shows that the initial reactive power output of all DG units is around 150 Var. When DG2 is disconnected at $t_{1}$, the reactive power outputs of DG1 and DG3 are increased to around 225 Var, which is reasonable because the total reactive power is 450 Var. When DG2 is re-connected to the microgrid at $t_{2}$, three DG units equally share the total reactive power again. Meanwhile, the unbalanced power sharing process during the plug-and-play is shown Fig. 8(b). It is clearly observed that unbalanced power is equally shared by three DG units during the stage 1 , and then are equally shared by DG 1 and DG3 due to the disconnection of the DG2. Finally, at $t_{2}$, when DG2 is plugged into the microgrid, the three DG units cooperatively share the unbalanced power.

Fig. 9 shows the power sharing performance under the load change. At $t<t_{1}$ (i.e., stage 1), all three DGs operate in the steady state and accurately share the reactive and unbalanced powers. At $t=t_{1}$, an extra $50 \Omega$ unbalanced load is connected to the microgrid. At $t=t_{2}$, this load is disconnected from the microgrid. As can be seen in Fig. 9, the control system efficiently handles the load change perfectly, and the reactive and unbalanced powers are accurately shared among three DGs all the time.

The proposed control methods are also studied under communication link failure conditions, as shown in Fig. 10. At $t<t_{1}$ (i.e., stage 1), the communication network is intact, and all three DG units share the same power. At $t=t_{1}$, the communication link between DG1 and DG2 is disconnected. Finally, at $t=t_{2}$, all the communication link failures are cleared. As seen from Fig. 11, despite the changes of the communication network, the power sharing performance are not affected at the steady state.

\section{B. Case-2: Nonlinear Load}

To verify the harmonic power sharing performance, a threephase diode rectifier load is connected to PCC. Before $t=t_{1}$ only the traditional droop method is adopted. The experimental results are presented in Fig. 12. As it can be observed, the traditional droop results in a poor power sharing. At $t=t_{1}$, when the proposed method is activated, the proposed control scheme enables DGs to share reactive and harmonic powers properly among each other.

Fig. 13(a) shows the phase-A of the output current of DGs when the traditional droop method is active. It is observed that there is a noticeable magnitude error among output currents of DGs, which implies there is a power sharing mismatch among them. With the activation of the proposed method, however, as shown in Fig. 13(b), the output currents of DGs are almost identical, which implies the unbalance and harmonic power are equally shared.

The plug-and-play capability of DGs in the presence of a nonlinear load is investigated in Fig. 14. The initial reactive power and harmonic power output of all DG units are around 100 Var and 155Var, when DG 2 is disconnected at $t_{1}$, the reactive and harmonic power output of DG1 and DG3 is around 150 Var and 233Var. Then, DG 2 is re-connected to the microgrid at $t_{2}$, the total 300 Var reactive power and 465 Var harmonic power are equally shared by these three DG units again.

The power sharing performance under the load change with nonlinear load is validated in Fig. 15. Similar to Fig. 8, the power can be accurately shared whenever a load is connected or disconnected from the microgrid.

The communication link failures with nonlinear loads are also studied in Fig. 16. The changes of the communication network are the same as in Fig. 9. Similar to the case of Fig. 10, even though the communication link changes, the power sharing performance is almost unaffected.

\section{Case-3: Comparison with Periodic Communication}

In this section, our proposed event-triggered control is compared to the periodic communication, where its sampling frequency is set as $f_{c}=1 \mathrm{kHz}$. The experimental results of harmonic power sharing with periodic communication is shown in Fig. 17. Compared to the proposed event-triggered control method in Fig. 12(b), they nearly achieve the same control performance. However, they are achieved at a different number of communication updates. The event-triggered time instant of DG1 harmonic power sharing is shown in Fig. 18. It is shown that the controller updates their communication in an aperiodic way. In addition, the communication triggering times of these two approaches for reactive power, unbalanced power and harmonic power sharing are calculated during $2 \mathrm{~s}$ period with the controllers activated, as shown in Fig. 19. From Fig. 19, it is concluded that the proposed event-triggered control method has few triggering times, which can highly reduce the communication burden among DG units.

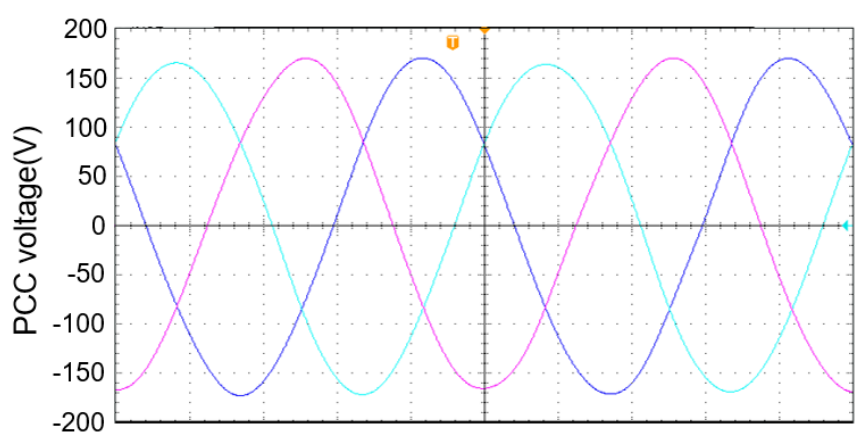

Fig. 5. PCC voltage with unbalanced loads 


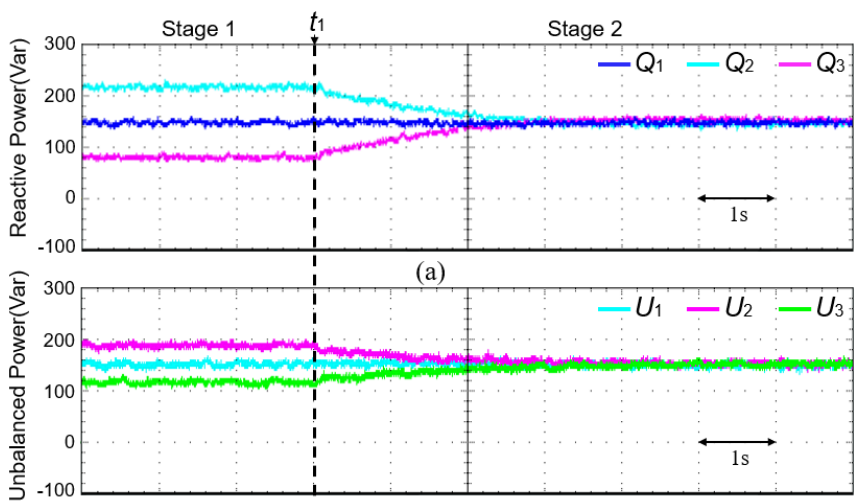

(b)

Fig. 6. Power sharing performance in the presence of unbalanced loads. (a) Reactive power. (b) Unbalanced power.

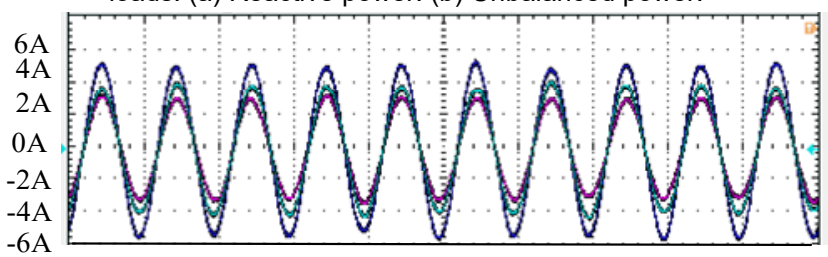

(a)

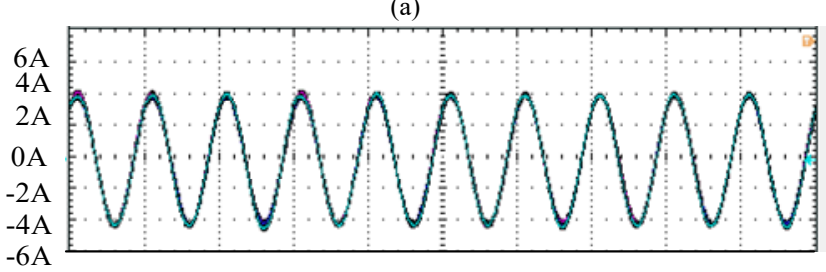

(b)

Fig. 7. Current performance with unbalanced loads. (a) Phase-A currents of DG units without the proposed method. (b) Phase-A currents of DG units with the proposed method(20ms/div).

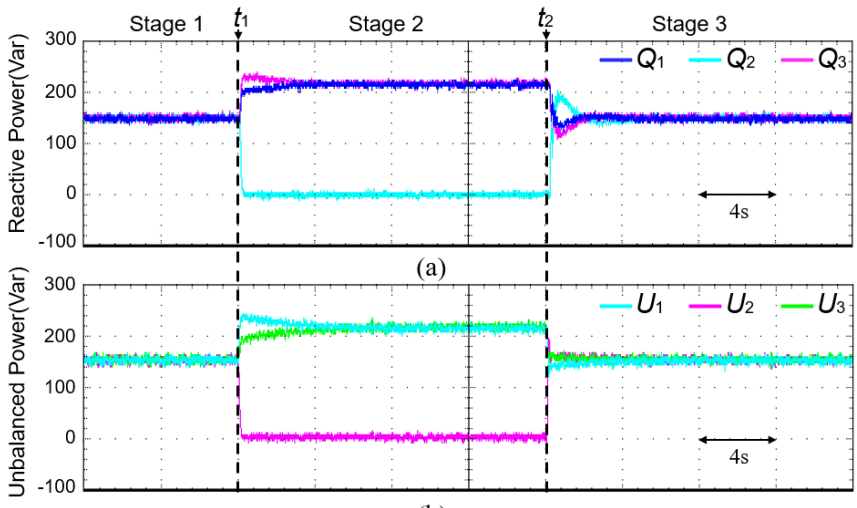

(b)

Fig. 8. Plug-and-play performance with unbalanced loads. (a) Reactive power. (b) Unbalanced power.

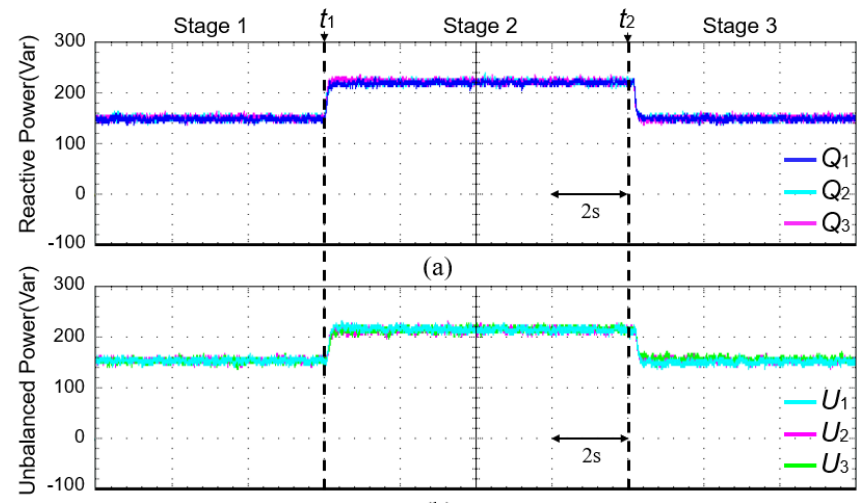

(b)

Fig. 9. Power sharing performance under unbalanced loads change. (a) Reactive power. (b) Unbalanced power.

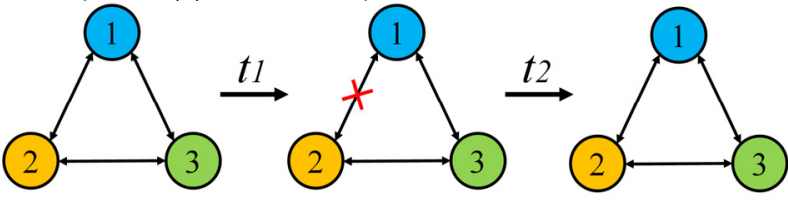

Fig. 10. Communication link failure process.

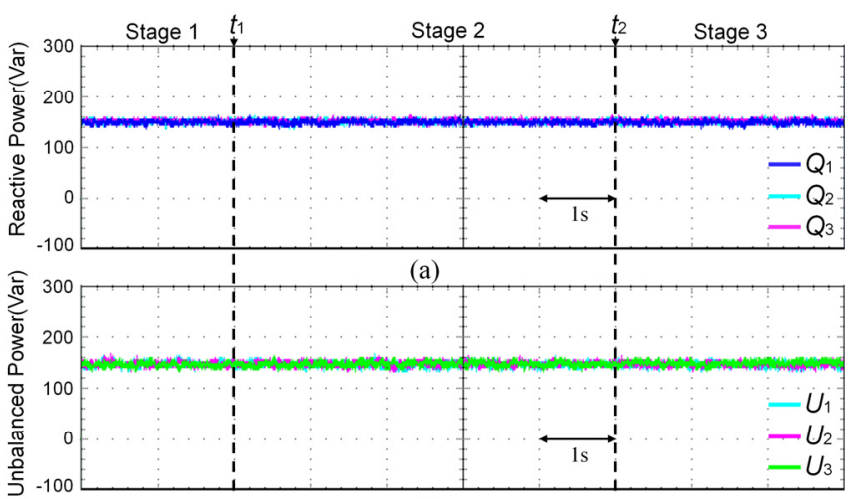

(b)

Fig. 11. Power sharing performance under communication link failure conditions. (a) Reactive power. (b) Unbalanced power.

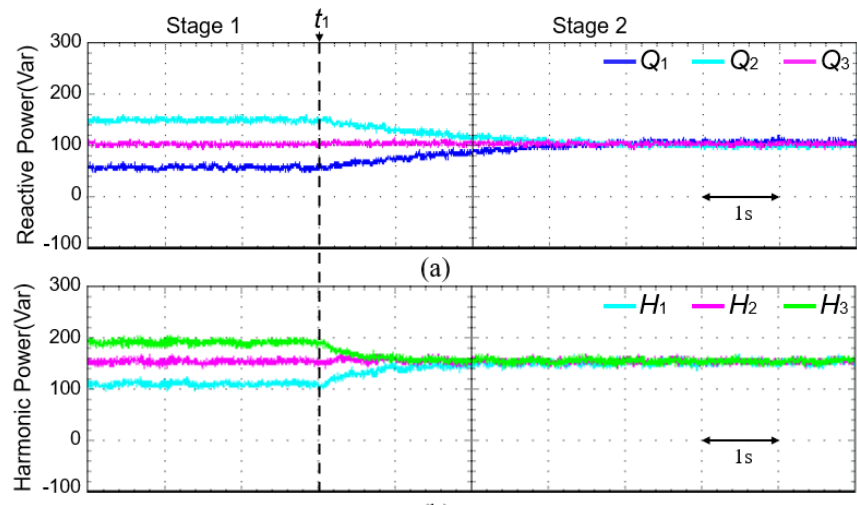

(b)

Fig. 12. Power sharing performance in the presence of the nonlinear load. (a) Reactive power. (b) Harmonic power. 


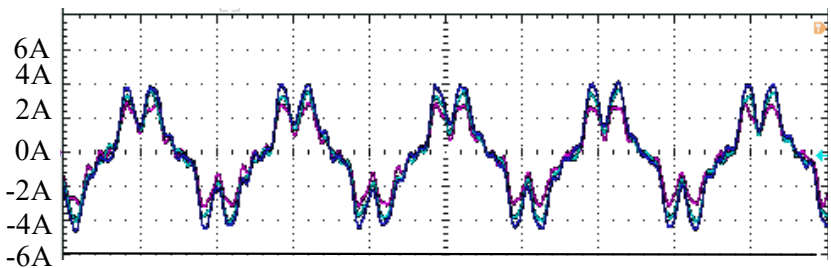

(a)

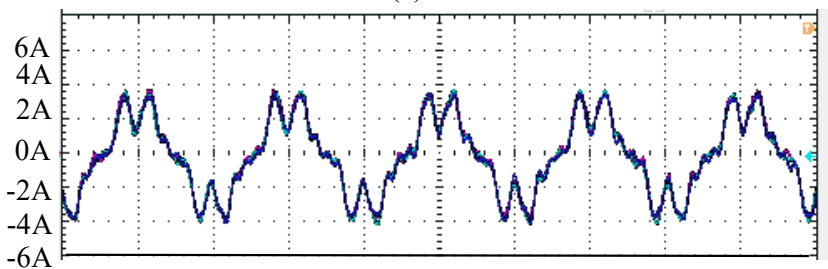

(b)

Fig. 13. Current sharing performance with nonlinear loads. (a) Phase-A currents of DG units without the proposed method. (b) Phase-A currents of DG units with the proposed method(10ms/div).

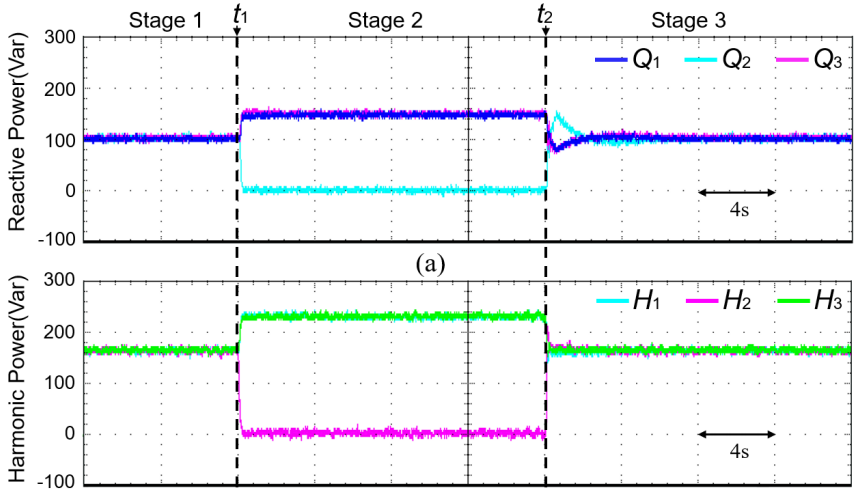

(b)

Fig. 14. Plug-and-play performance with nonlinear loads. (a) Reactive power. (b) Harmonic power.

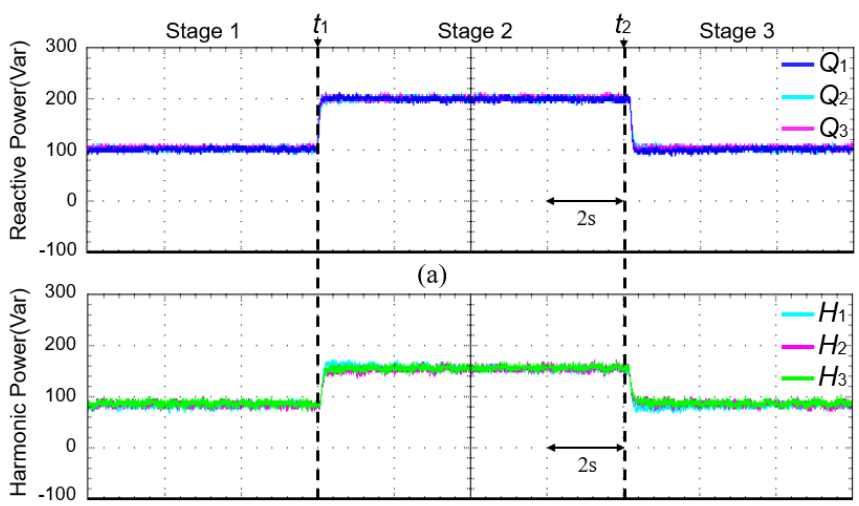

(b)

Fig. 15. Power sharing performance under nonlinear loads change. (a) Reactive power. (b) Harmonic power.

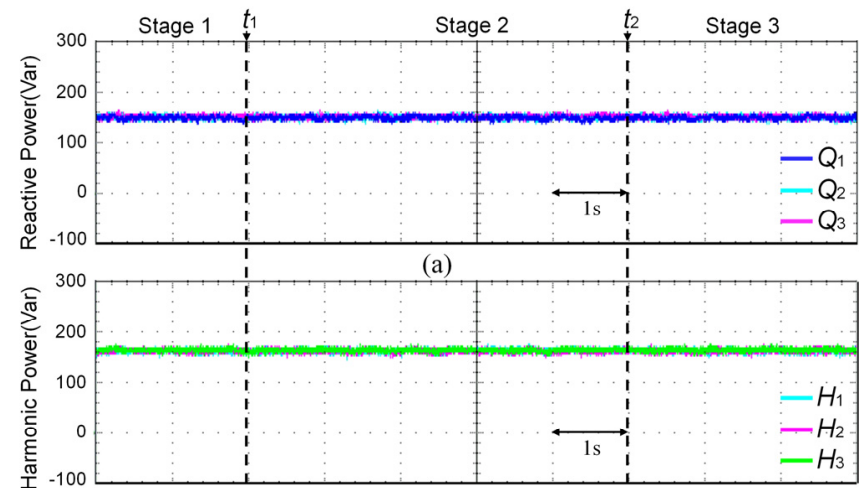

(b)

Fig. 16. Power sharing performance under communication link failure conditions. (a) Reactive power. (b) Harmonic power.

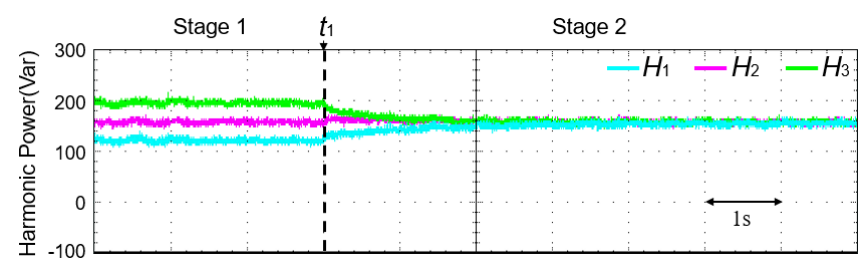

Fig. 17. Harmonic power sharing performance with nonlinear loads in continuous communication way.

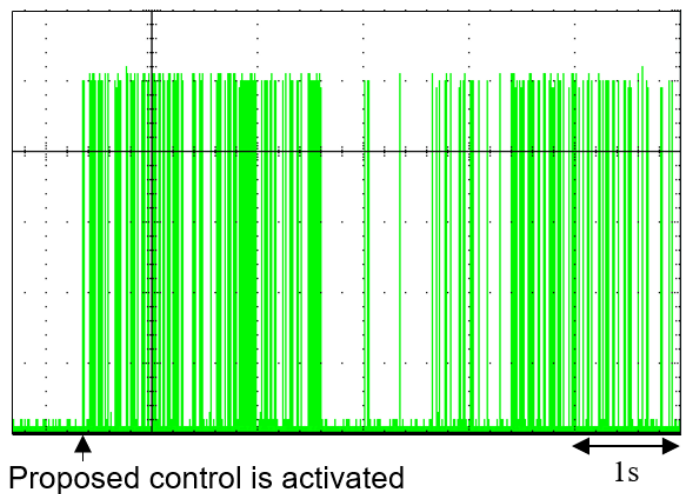

Fig.18. Event-triggered time instant with DG1 harmonic power sharing.

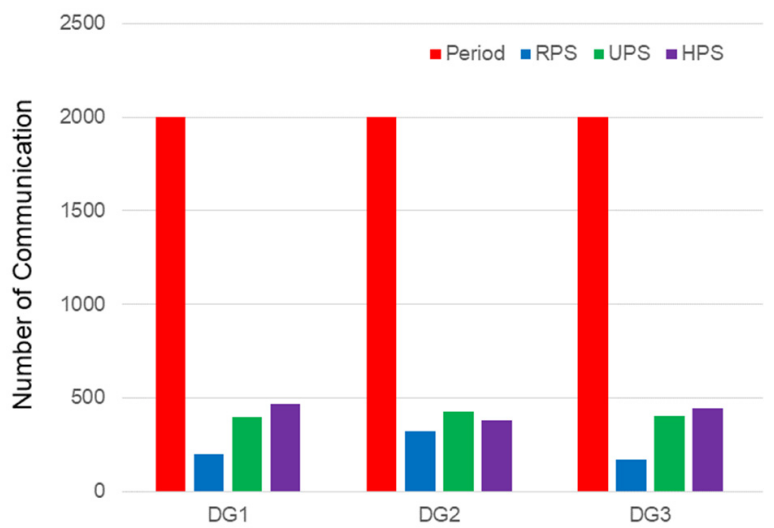

Fig. 19. Comparison for the number of communication. (RPS: reactive power sharing, UPS: unbalanced power sharing, HPS: harmonic power sharing).

\section{CONCLUSION}

This paper proposed an event-triggered distributed control 
strategy for the reactive, unbalance and harmonic power sharing in an islanded microgrid. Compared with the periodically distributed method, the proposed control strategy could realize the accurate power sharing while highly reducing communication data exchange, and achieving the plug-and-play feature among the DG units. The stability of the proposed control strategy was proved with Lyapunov function and the Zeno behavior can be excluded. Experimental results from microgrid laboratory demonstrated the effectiveness of the proposed scheme.

\section{REFERENCES}

[1] J. M. Carrasco et al., "Power-Electronic Systems for the Grid Integration of Renewable Energy Sources: A Survey," Ieee T Ind Electron, vol. 53, no. 4, pp. 1002-1016, 2006, doi: 10.1109/TIE.2006.878356.

[2] J. M. Guerrero, J. C. Vasquez, J. Matas, L. G. de Vicuna, and M. Castilla, "Hierarchical Control of Droop-Controlled AC and DC Microgrids-A General Approach Toward Standardization," (in English), Ieee Transactions on Industrial Electronics, Article vol. 58, no. 1, pp. 158-172, Jan 2011, doi: 10.1109/tie.2010.2066534.

[3] J. C. Vasquez, J. M. Guerrero, A. Luna, P. Rodriguez, and R. Teodorescu, "Adaptive Droop Control Applied to Voltage-Source Inverters Operating in Grid-Connected and Islanded Modes," IEEE Transactions on Industrial Electronics, vol. 56, no. 10, pp. 4088-4096, 2009, doi: 10.1109/TIE.2009.2027921.

[4] K. D. Brabandere, B. Bolsens, J. V. d. Keybus, A. Woyte, J. Driesen, and R. Belmans, "A Voltage and Frequency Droop Control Method for Parallel Inverters," IEEE Transactions on Power Electronics, vol. 22, no. 4, pp. 1107-1115, 2007.

[5] J. C. Vasquez, R. A. Mastromauro, J. M. Guerrero, and M. Liserre, "Voltage Support Provided by a Droop-Controlled Multifunctional Inverter," IEEE Transactions on Industrial Electronics, vol. 56, no. 11, pp. 4510-4519, 2009.

[6] J. Rocabert, A. Luna, F. Blaabjerg, and P. Rodríguez, "Control of Power Converters in AC Microgrids," IEEE Transactions on Power Electronics, vol. 27 , no. 11, pp. 4734-4749, 2012.

[7] H. Mahmood, D. Michaelson, and J. Jiang, "Reactive Power Sharing in Islanded Microgrids Using Adaptive Voltage Droop Control," IEEE Transactions on Smart Grid, vol. 6, no. 6, pp. 3052-3060, 2015.

[8] J. He and Y. W. Li, "An Enhanced Microgrid Load Demand Sharing Strategy," IEEE Transactions on Power Electronics, vol. 27, no. 9, pp. 3984-3995, 2012.

[9] X. Hou, Y. Sun, H. Han, Z. Liu, W. Yuan, and M. Su, "A fully decentralized control of grid-connected cascaded inverters," IEEE Transactions on Sustainable Energy, vol. 10, no. 1, pp. 315-317, 2019, doi: 10.1109/TPWRD.2018.2816813.

[10] T. Vandoorn, B. Meersman, J. De Kooning, and L. Vandevelde, "Controllable Harmonic Current Sharing in Islanded Microgrids: DG Units With Programmable Resistive Behavior Toward Harmonics," Ieee $T$ Power Deliver, vol. 27, no. 2, pp. 831-841, 2012, doi: 10.1109/tpwrd.2011.2176756.

[11] D. De and V. Ramanarayanan, "Decentralized Parallel Operation of Inverters Sharing Unbalanced and Nonlinear Loads," Ieee T Power Electr, vol. 25 , no. 12, pp. 3015-3025, 2010, doi: 10.1109/TPEL.2010.2068313.

[12] P. Sreekumar and V. Khadkikar, "A New Virtual Harmonic Impedance Scheme for Harmonic Power Sharing in an Islanded Microgrid," Ieee T Power Deliver, vol. 31, no. 3, pp. 936-945, 2016, doi: 10.1109/tpwrd.2015.2402434.

[13] B. Liu, Z. Liu, J. Liu, R. An, H. Zheng, and Y. Shi, "An Adaptive Virtual Impedance Control Scheme Based on Small-AC-Signal Injection for Unbalanced and Harmonic Power Sharing in Islanded Microgrids," Ieee T Power Electr, vol. 34, no. 12, pp. 12333-12355, 2019, doi: 10.1109/tpel.2019.2905588.

[14] Y. Han, P. Shen, X. Zhao, and J. M. Guerrero, "An Enhanced Power Sharing Scheme for Voltage Unbalance and Harmonics Compensation in an Islanded AC Microgrid," IEEE Transactions on Energy Conversion, vol. 31, no. 3, pp. 1037-1050, 2016, doi: 10.1109/tec.2016.2552497.

[15] J. Zhou, S. Kim, H. Zhang, Q. Sun, and R. Han, "Consensus-Based Distributed Control for Accurate Reactive, Harmonic, and Imbalance
Power Sharing in Microgrids," IEEE Transactions on Smart Grid, vol. 9, no. 4, pp. 2453-2467, 2018, doi: 10.1109/TSG.2016.2613143.

[16] H. Jinwei and L. Yun Wei, "An Enhanced Microgrid Load Demand Sharing Strategy," Power Electronics, IEEE Transactions on, vol. 27, no. 9, pp. 3984-3995, 2012, doi: 10.1109/TPEL.2012.2190099.

[17] H. Jinwei, L. Yun Wei, and F. Blaabjerg, "An Enhanced Islanding Microgrid Reactive Power, Imbalance Power, and Harmonic Power Sharing Scheme," Power Electronics, IEEE Transactions on, vol. 30, no. 6, pp. 3389-3401, 2015, doi: 10.1109/TPEL.2014.2332998.

[18] J. Lu et al., "DC-Link Protection and Control in Modular Uninterruptible Power Supply," Ieee T Ind Electron, vol. 65, no. 5, pp. 3942-3953, 2018, doi: 10.1109/TIE.2017.2760847.

[19] L.-Y. Lu and C.-C. Chu, "Consensus-Based Droop Control Synthesis for Multiple DICs in Isolated Micro-Grids," IEEE Transactions on Power Systems, vol. 30, no. 5, pp. 2243-2256, 2015, doi: 10.1109/tpwrs.2014.2368135.

[20] H. Zhang, S. Kim, Q. Sun, and J. Zhou, "Distributed Adaptive Virtual Impedance Control for Accurate Reactive Power Sharing Based on Consensus Control in Microgrids," IEEE Transactions on Smart Grid, vol. 8, no. 4, pp. 1749-1761, 2017, doi: 10.1109/TSG.2015.2506760.

[21] L. Meng et al., "Distributed Voltage Unbalance Compensation in Islanded Microgrids by Using a Dynamic Consensus Algorithm," IEEE Transactions on Power Electronics, vol. 31, no. 1, pp. 827-838, 2016, doi: 10.1109/TPEL.2015.2408367.

[22] L. Ding, Q.-L. Han, and X.-M. Zhang, "Distributed Secondary Control for Active Power Sharing and Frequency Regulation in Islanded Microgrids Using an Event-Triggered Communication Mechanism," Ieee T Ind Inform, vol. 15 , no. 7 , pp. 3910-3922, 2019, doi: 10.1109/tii.2018.2884494.

[23] L. Ding, Q.-L. Han, X. Ge, and X.-M. Zhang, "An Overview of Recent Advances in Event-Triggered Consensus of Multiagent Systems," IEEE Transactions on Cybernetics, vol. 48, no. 4, pp. 1110-1123, 2018, doi: 10.1109/tcyb.2017.2771560.

[24] T. Qian, Y. Liu, W. Zhang, W. Tang, and M. Shahidehpour, "EventTriggered Updating Method in Centralized and Distributed Secondary Controls for Islanded Microgrid Restoration," IEEE Transactions on Smart Grid, vol. 11, no. 2, pp. 1387-1395, 2020, doi: 10.1109/TSG.2019.2937366.

[25] B. Abdolmaleki, Q. Shafiee, A. R. Seifi, M. M. Arefi, and F. Blaabjerg, "A Zeno-Free Event-Triggered Secondary Control for AC Microgrids," IEEE Transactions on Smart Grid, vol. 11, no. 3, pp. 1905-1916, 2020, doi: 10.1109/TSG.2019.2945250.

[26] M. Chen, X. Xiao, and J. M. Guerrero, "Secondary Restoration Control of Islanded Microgrids With a Decentralized Event-Triggered Strategy," IEEE Transactions on Industrial Informatics, vol. 14, no. 9, pp. 38703880, 2018.

[27] Y. Fan, G. Hu, and M. Egerstedt, "Distributed Reactive Power Sharing Control for Microgrids With Event-Triggered Communication," Ieee T Contr Syst T, vol. 25, no. 1, pp. 118-128, 2017.

[28] L. Ding, Q. Han, X. Ge, and X. Zhang, "An Overview of Recent Advances in Event-Triggered Consensus of Multiagent Systems," IEEE Transactions on Cybernetics, vol. 48, no. 4, pp. 1110-1123, 2018, doi: 10.1109/TCYB.2017.2771560.

[29] C. Li, X. Yu, W. Yu, T. Huang, and Z.-W. Liu, "Distributed EventTriggered Scheme for Economic Dispatch in Smart Grids," Ieee T Ind Inform, vol. 12, no. 5, pp. 1775-1785, 2016, doi: 10.1109/tii.2015.2479558.

[30] J. He, Y. W. Li, and F. Blaabjerg, "An Enhanced Islanding Microgrid Reactive Power, Imbalance Power, and Harmonic Power Sharing Scheme," IEEE Transactions on Power Electronics, vol. 30, no. 6, pp. 3389-3401, 2015, doi: 10.1109/TPEL.2014.2332998.

[31] Y. Su and J. Huang, "Cooperative Output Regulation of Linear MultiAgent Systems," IEEE Transactions on Automatic Control, vol. 57, no. 4, pp. 1062-1066, 2012.

[32] P. Rodríguez, A. Luna, I. Candela, R. Mujal, R. Teodorescu, and F. Blaabjerg, "Multiresonant Frequency-Locked Loop for Grid Synchronization of Power Converters Under Distorted Grid Conditions," IEEE Transactions on Industrial Electronics, vol. 58, no. 1, pp. 127-138, 2011, doi: 10.1109/TIE.2010.2042420.

[33] D. V. Dimarogonas, E. Frazzoli, and K. H. Johansson, "Distributed EventTriggered Control for Multi-Agent Systems," IEEE Transactions on Automatic Control, vol. 57, no. 5, pp. 1291-1297, 2012. 
[34] C. Nowzari and J. Cortés, "Zeno-free, distributed event-triggered communication and control for multi-agent average consensus," in 2014 American Control Conference, 4-6 June 2014 2014, pp. 2148-2153.

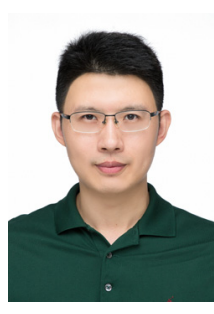

Jinghang Lu (S'14-M'18) received the B.Sc. degree in electrical engineering from Harbin Institute of Technology, China, in 2009, two M.Sc. degrees both in electrical engineering from Harbin Institute of Technology, China, in 2011, and University of Alberta, Canada, in 2014, respectively, and the Ph.D. degree in Power Electronics from Aalborg University, Aalborg, Denmark, in 2018. From 2018 to 2019, He was a Research Fellow in Nanyang Technological University, Singapore. Currently, he is an Assistant Professor with Harbin Institute of Technology (Shenzhen), Shenzhen, China

His research interests include uninterruptible power supply, distributed control of microgrids, and control of power converters.

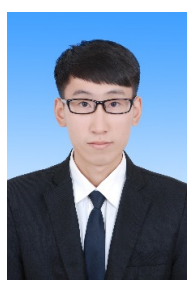

Ming Zhao received the B.E. degree in electrical engineering and automation from Yanshan University, Qinhuangdao, China in 2017. He is currently pursuing the master degree in electrical engineering from Harbin Institute of Technology (Shenzhen), China. His research interests include event-triggered mechanisms and microgrid.

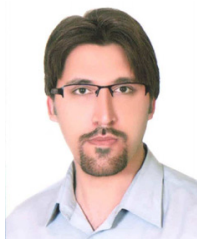

Saeed Golestan (sgd@et.aau.dk) received a B.Sc. degree from Shahid Chamran University of Ahvaz, Iran, in 2006 , an M.Sc. degree from the Amirkabir University of Technology, Tehran, Iran, in 2009, and a PhD degree from Aalborg University, Aalborg, Denmark, in 2018, all in electrical engineering. $\mathrm{He}$ is currently an assistant professor at the Department of Energy Technology, Aalborg University, Aalborg, Denmark. His research interests include modeling, synchronization, and control of power electronic systems and microgrids. He is an IEEE senior member, and an associate editor of IEEE Transactions on Power Electronics.

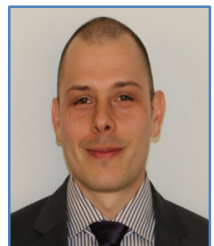

Tomislav Dragičević (S'09-M'13-SM'17) received the M.Sc. and the industrial Ph.D. degrees in Electrical Engineering from the Faculty of Electrical Engineering, University of Zagreb, Croatia, in 2009 and 2013 respectively. From 2013 until 2016 he has been a Postdoctoral researcher at Aalborg University, Denmark. From 2016 until 2020 he was an Associate Professor at Aalborg University, Denmark. From 2020 he is a Professor at the Technical University of Denmark. He made a guest professor stay at Nottingham University, UK during spring/summer of 2018. His research interest is application of advanced control, optimization and artificial intelligence inspired techniques to provide innovative and effective solutions to emerging challenges in design, control and cyber-security of power electronics intensive electrical distributions systems and microgrids. He has authored and co-authored more than 250 technical publications (more than 120 of them are published in international journals, mostly in IEEE), 8 book chapters and a book in the field.

$\mathrm{He}$ serves as an Associate Editor in the IEEE TRANSACTIONS ON INDUSTRIAL ELECTRONICS, in IEEE TRANSACTIONS ON POWER ELECTRONICS, in IEEE Emerging and Selected Topics in Power Electronics and in IEEE Industrial Electronics Magazine. Dr. Dragičević is a recipient of the Končar prize for the best industrial $\mathrm{PhD}$ thesis in Croatia, a Robert Mayer Energy Conservation award, and he is a winner of an Alexander von Humboldt fellowship for experienced researchers.

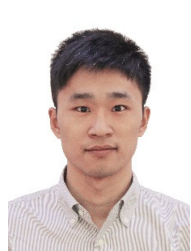

Xuewei Pan (S'12-M'16) received the B.E. degree in Electronic Engineering from University of Electronic Science and Technology of China (UESTC), Chengdu, China in 2011. He obtained his Ph.D. degree in the area of Power Electronics at Electrical and Computer Engineering, National University of Singapore in 2015. He worked as a research fellow in Energy Research Institute @ NTU (ERI@N) from July 2014 to September 2016 in Singapore.
Currently He is an Associate Professor in Harbin Institute of Technology, Shenzhen, China. His research interests include distributed generation, renewable integration, micro-grid energy system, soft-switching methods and modulation techniques for high frequency power conversion

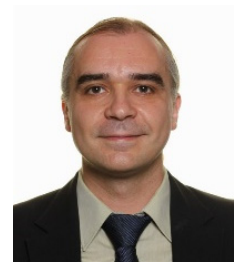

Josep M. Guerrero (S'01-M'04-SM'08-FM'15) received the B.S. degree in telecommunications engineering, the M.S. degree in electronics engineering, and the Ph.D. degree in power electronics from the Technical University of Catalonia, Barcelona, in 1997, 2000 and 2003, respectively. Since 2011, he has been a Full Professor with the Department of Energy Technology, Aalborg University, Denmark, where he is responsible for the Microgrid Research Program. From 2014 he is chair Professor in Shandong University; from 2015 he is a distinguished guest Professor in Hunan University; and from 2016 he is a visiting professor fellow at Aston University, UK, and a guest Professor at the Nanjing University of Posts and Telecommunications. J. M. Guerrero was supported by VILLUM FONDEN under the VILLUM Investigator Grant (no. 25920): Center for Research on Microgrids (CROM);www.crom.et.aau.dk

His research interests is oriented to different microgrid aspects, including power electronics, distributed energy-storage systems, hierarchical and cooperative control, energy management systems, smart metering and the internet of things for $\mathrm{AC} / \mathrm{DC}$ microgrid clusters and islanded minigrids; recently specially focused on maritime microgrids for electrical ships, vessels, ferries and seaports. Prof. Guerrero is an Associate Editor for a number of IEEE TRANSACTIONS. He has published more than 450 journal papers in the fields of microgrids and renewable energy systems, which are cited more than 30,000 times. He received the best paper award of the IEEE Transactions on Energy Conversion for the period 2014-2015, and the best paper prize of IEEE-PES in 2015. As well, he received the best paper award of the Journal of Power Electronics in 2016. During five consecutive years, from 2014 to 2018, he was awarded by Thomson Reuters as Highly Cited Researcher. In 2015 he was elevated as IEEE Fellow for his contributions on "distributed power systems and microgrids." 